\title{
Microscopic Model of the Optical Absorption of Carbon Nanotubes Functionalized with Molecular Spiropyran Photoswitches
}

\author{
E. Malic,,${ }^{1, *}$ C. Weber, ${ }^{1}$ M. Richter, ${ }^{2}$ V. Atalla, ${ }^{3}$ T. Klamroth, ${ }^{4}$ P. Saalfrank, ${ }^{4}$ S. Reich,,${ }^{5}$ and A. Knorr ${ }^{1}$ \\ ${ }^{1}$ Institut für Theoretische Physik, Technische Universität Berlin, 10623 Berlin, Germany \\ ${ }^{2}$ Department of Chemistry, University of California Irvine, Irvine, California 92697-2025, USA \\ ${ }^{3}$ Fritz-Haber-Institut der Max-Planck-Gesellschaft, Faradayweg 4-6, 14195 Berlin, Germany \\ ${ }^{4}$ Institut für Chemie, Universität Potsdam, Karl-Liebknecht-Strasse 24-25, 14476 Potsdam-Golm, Germany \\ ${ }^{5}$ Fachbereich Physik, Freie Universität Berlin, 14195 Berlin, Germany
}

(Received 13 October 2010; published 1 March 2011)

\begin{abstract}
The adsorption of molecules to the surface of carbon nanostructures opens a new field of hybrid systems with distinct and controllable properties. We present a microscopic study of the optical absorption in carbon nanotubes functionalized with molecular spiropyran photoswitches. The switching process induces a change in the dipole moment leading to a significant coupling to the charge carriers in the nanotube. As a result, the absorption spectra of functionalized tubes reveal a considerable redshift of transition energies depending on the switching state of the spiropyran molecule. Our results suggest that carbon nanotubes are excellent substrates for the optical readout of spiropyran-based molecular switches. The gained insights can be applied to other noncovalently functionalized one-dimensional nanostructures in an externally induced dipole field.
\end{abstract}

DOI: 10.1103/PhysRevLett.106.097401

PACS numbers: 78.67.Ch, 42.79.Ta, 71.35.Cc, 78.40.Ri

Single-walled carbon nanotubes (CNTs) consisting of a single layer of carbon atoms show a high sensitivity to changes in their surrounding medium, making them good candidates for sensitive nanoscale detectors [1-3]. They furthermore present ideal substrates for the functionalization with molecules $[1,4]$. Depending on their chiral angle and diameter, they offer a variety of substrates with different optical and electronic properties including metallic and semiconducting structures with a large range of band gaps [1]. The functionalization of nanotubes enables the control and optimization of their properties and is a promising strategy to exploit their tremendous application potential [1-6]. In particular, the functionalization with photochrome molecules is promising for engineering switches on the molecular level [5-8] and incorporating them into solid-state technologies [9]. An example for such molecules are spiropyrans, which can be reversibly switched between two different conformations: the planar merocyanine (MC) and the orthogonal spiropyran (SP) [Fig. 1(a)]. The conversion is induced by visible and ultraviolet light. It is accompanied by a significant change in the molecular dipole moment [9]. As a result, the attached molecule has-depending on its conformation-a unique influence on the carrier mobility and the optical transitions in the substrate CNT. Therefore, we expect a significant change of the electronic and optical properties of the CNT induced by the molecular switching process.

In this Letter, we present a microscopic study of the optical properties of pristine and noncovalently spiropyran-functionalized carbon nanotubes. A better understanding of their optical properties is of crucial importance for the realization of efficient molecular switches.
Our microscopic investigations offer new insights, which can guide future optical experiments. The coupling of the carriers in the CNT to the externally induced dipole fields depends on the dipole moment, its orientation with respect to the CNT axis, the molecular dipole density, and the distribution of molecules along the CNT surface [Fig. 1(b)]. Our aim is a thorough understanding of the functionalization parameters and their influence on the absorption spectra of CNTs. In particular, it is of fundamental interest to address the question whether CNTs are suitable substrates for an unambiguous optical readout of the spiropyran-based molecular photoswitches.
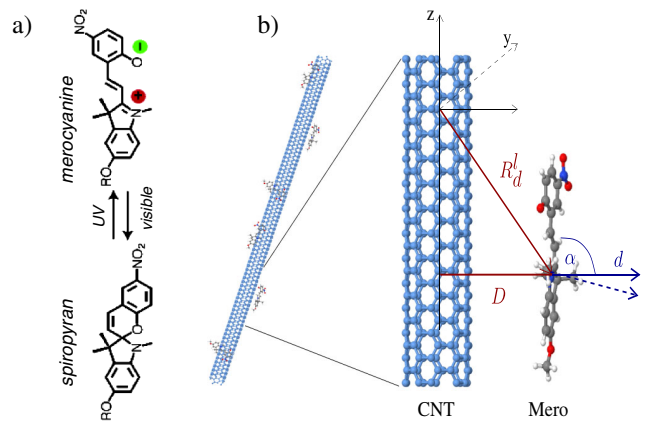

FIG. 1 (color online). (a) The switching process between the merocyanine (MC) and the spiropyran (SP) conformation is induced by visible light, while backswitching is initiated by ultraviolet light (after Ref. [6]). (b) Merocyanine-functionalized exemplary $(10,0)$ nanotube with the molecular dipole moment $d$ and its orientation $\alpha$ with respect to the CNT axis. The solid (dashed, blue) arrow shows the mero (spiro) dipole orientation predicted by density-functional theory calculations. 
In order to describe the optical and electronic properties of functionalized carbon nanotubes, we apply the formalism of density matrix theory, an established method for microscopic description of many-particle systems [10,11]. The modeled situation corresponds to pristine CNTs located in a static dipole field induced by noncovalently adsorbed spiropyran molecules. In contrast to covalent functionalization, the van der Waals interaction is less invasive and only slightly changes the electronic structure of the tube [4]. To obtain a realistic description of the adsorbtion of molecules including their position on the CNT surface and the resulting dipole fields, densityfunctional theory calculations have been performed. The obtained results are used as input parameters for the Bloch equations. The dipole moments of the isolated molecules are determined for optimized geometries at the B3LYP/6-31G* level of theory as implemented in Gaussian 09 [12].

The absorption coefficient $\alpha(\omega) \propto \omega \operatorname{Im} \chi(\omega)$ is given by the optical susceptibility [13-15] $\chi(\omega) \propto$ $-i \sum_{k} \operatorname{Re}\left[M_{v c}^{z}(k) \sigma_{k k}^{v c}(\omega)\right] /\left[\omega^{2} A(\omega)\right]$, which is the linear response function of the perturbed system. For a twoband system, $\chi(\omega)$ is determined by the Fourier transform of the microscopic polarization $\sigma_{k k}^{v c}(\omega)$, the optical matrix element $M_{v c}^{z}(k)$ along the nanotube axis [13], and the applied vector potential $A(\omega)$ of the optical field. The microscopic polarization $\sigma_{k k}^{v c}(t)=\left\langle a_{v k}^{+} a_{c k}\right\rangle(t)$ is a measure for the transition probability of an electron between the two states $|v k\rangle$ and $|c k\rangle$. Here $k$ denotes the wave vector and $v$, $c$ the valence and the conduction band, respectively. The starting point to derive $\chi(\omega)$ is the Hamilton operator $H=$ $H_{0}+H_{c-l}+H_{c-c}+H_{c-d}$ including the noninteracting electronic contribution $H_{0}$, the electron-light coupling $H_{c-l}$, the Coulomb interaction $H_{c-c}$, and the CNT-dipole interaction $H_{c-d}$. It determines the temporal dynamics of an observable within the Heisenberg equation of motion [10] yielding the microscopic many-particle Bloch equation for the CNT-molecule hybrid structure

$$
\begin{aligned}
\dot{\sigma}_{k_{1} k_{2}}^{v c}= & -i\left(\tilde{\omega}_{k_{1} k_{2}}-i \gamma\right) \sigma_{k_{1} k_{2}}^{v c}+i \tilde{\Omega}_{k_{1} k_{2}} \\
& -\frac{i}{\hbar} \sum_{k^{\prime}}\left(g_{k_{2} k^{\prime}}^{c c} \sigma_{k_{1} k^{\prime}}^{v c}-g_{k^{\prime} k_{1}}^{v v} \sigma_{k^{\prime} k_{2}}^{v c}\right) .
\end{aligned}
$$

The equation describes the dynamics of the dipole-induced transitions $\sigma_{k_{1} k_{2}}^{v c}(t)=\left\langle a_{v k_{1}}^{+} a_{c k_{2}}\right\rangle(t)$ with a momentum transfer $\left(k_{1}-k_{2}\right) \neq 0$ characterizing the spatially inhomogeneous situation. In particular, it contains the microscopic polarization $\sigma_{k k}^{v c}$ in the limiting case of $k_{1}=k_{2}$, which has a predominant influence on the dynamics of $\sigma_{k_{1} k_{2}}^{v c}(t)$. Equation (2) has been derived assuming linear optics, where the driving field is considered to be small resulting in a negligible change of the occupation in the valence and conduction band [10].

The Coulomb interaction is considered within the Hartree-Fock level leading to (i) the renormalization of the Rabi frequency $\tilde{\Omega}_{k_{1} k_{2}}(t)=\frac{e_{0}}{m_{0}} M_{c v}^{z}\left(k_{2}\right) A(t) \delta_{k_{1}, k_{2}}-$ $\frac{1}{\hbar} \sum_{k^{\prime}} V_{e-h}\left(k^{\prime}, k_{1}, k_{2}\right) p_{k^{\prime}}(t)$ due to the attractive electronhole interaction $V_{e-h}\left(k^{\prime}, k_{1}, k_{2}\right)$, which describes the formation of excitons $[14,15]$ and (ii) the renormalization of the band gap $\tilde{\omega}_{k_{1} k_{2}}=\left(\omega_{c k_{2}}-\omega_{v k_{1}}\right)-\frac{1}{\hbar} \sum_{k^{\prime}} V_{e-e}\left(k^{\prime}, k_{1}, k_{2}\right)$ due to the repulsive electron-electron interaction $V_{e-e}\left(k^{\prime}, k_{1}, k_{2}\right)$, which corresponds to the self-energy correction. The Coulomb matrix elements are calculated within the tight-binding (TB) approximation and a regularized Coulomb potential (Ohno parametrization) $[15,16]$. The parameter $\gamma$ in Eq. (2) describes the dephasing of the microscopic polarization by scattering contributions beyond the Hartree-Fock level. It determines the peak width in optical spectra, but has no influence on the position.

The strength of the CNT-molecule interaction is given by the matrix element $g_{k_{1} k_{2}}^{\lambda \lambda^{\prime}}=\left\langle\Psi_{\lambda, k_{1}}\left|\sum_{l} e_{0} \phi_{d}^{l}(\boldsymbol{r})\right| \Psi_{\lambda^{\prime}, k_{2}}\right\rangle$ with the zone-folded TB wave functions $\Psi_{\lambda, k}(\boldsymbol{r})$ and the dipole potential $\phi_{d}^{l}(\boldsymbol{r})=\frac{1}{4 \pi \varepsilon_{0}} \frac{\boldsymbol{d} \cdot\left(\boldsymbol{r}-\boldsymbol{R}_{d}^{l}\right)}{\left|\boldsymbol{r}-\boldsymbol{R}_{d}^{l}\right|^{3}}$ induced by the molecule $l$ at the position $\boldsymbol{R}_{d}^{l}$. The coupling element can be partially evaluated analytically by inserting TB wave functions with effective $p_{z}$-hydrogen orbitals [14]. The coupling element does not depend on the length of the introduced supercell, but on the molecular dipole density $n=N_{s} / L_{s}$ corresponding to the number of molecules $N_{s}$ per supercell length $L_{s}$.

Figure 2 shows the intraband matrix element $g_{k k^{\prime}}^{\lambda \lambda}$ as a function of the momentum transfer $k$ (for an exemplary $k^{\prime}=0$ ) for different situations. Depending on the orientation of the dipole (rotation $\alpha$ within the CNT-molecule plane [Fig. 1(b)]), the matrix element shows a different behavior, as illustrated in Fig. 2(a). For dipoles with a parallel orientation $\alpha=0^{\circ}$ with respect to the CNT axis, we observe that the coupling vanishes for $k=0$, i.e., for processes with no momentum transfer. Then, the interaction is enhanced with increasing $k$ up to a maximal value at $k \approx 0.7 \mathrm{~nm}^{-1}$ followed by an exponential-like decrease. For the perpendicular orientation $\alpha=90^{\circ}$, in contrast, the coupling reaches maximal values for small momentum transfers $k \approx 0$. This behavior can be traced back to the dumbbell-like shape of the dipole potential.

Increasing the dipole density, we observe the formation of a multiple-peak structure in the matrix element [Fig. 2(b)]. This stems from the interference effects at the CNT surface due to dipole contributions from different molecules. The separation of the peaks is given by the inverse distance between two molecules. As a result, for small dipole densities, we obtain an increasing number of peaks. In the limiting case of only one molecule per supercell, the interference effects completely vanish [Fig. 2(a)]. Furthermore, it is important whether the molecules are aligned periodically or randomly along the CNT [Fig. 2(c)]. In the latter case, the clear multipeak structure of the matrix element is smeared to a certain extent. 


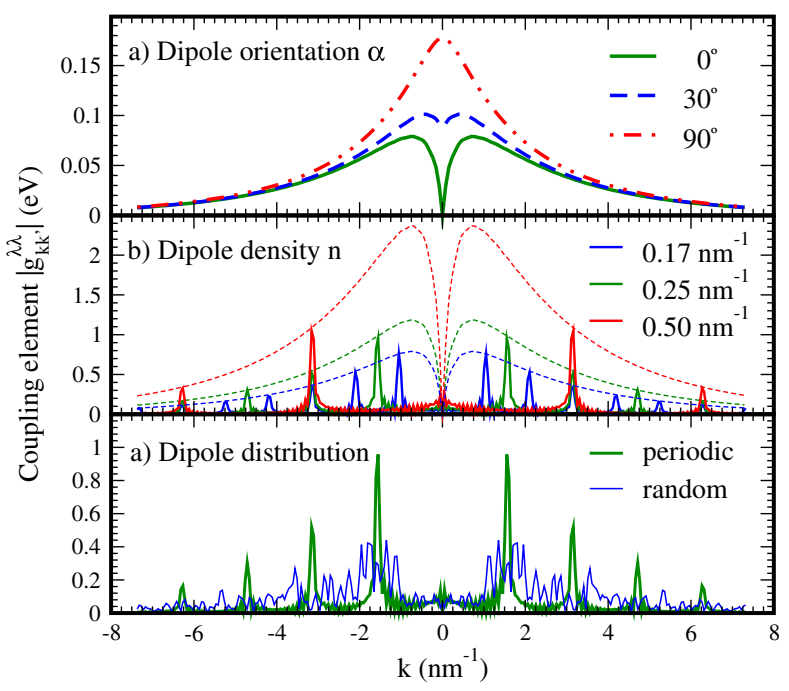

FIG. 2 (color online). CNT-dipole matrix element $g_{k k^{\prime}}^{\lambda \lambda}$ as a function of the momentum transfer $k$ (for an exemplary $k^{\prime}=0$ ) (a) for different orientations $\alpha$ with respect to the CNT axis (at a constant very small dipole density $n=0.017 \mathrm{~nm}^{-1}$ ), (b) for different dipole densities $n$ (at a constant $\alpha=0^{\circ}$ ), and (c) for different dipole distributions (at constant $\alpha=0^{\circ}$ and $n=$ $0.25 \mathrm{~nm}^{-1}$ ). The matrix elements exhibit a multipeak structure for larger dipole densities due to the interference of different molecules. The dashed line in (b) shows the corresponding envelope functions.

Now we have all the ingredients to calculate the absorption coefficient $\alpha(\omega)$, by evaluating the many-particle Bloch equations for the CNT-molecule hybrid structure [Eq. (2)]. We investigate the changes in the absorption spectrum of the exemplary $(10,0)$ nanotube, which are induced by the noncovalently attached spiropyran molecule. Since excitonic effects in CNTs are known to be strongly dependent on the environment [17], we investigate both excitonic and free-particle absorption spectra. The latter appropriately describe CNTs in media with a large dielectric background constant or tubes with a large diameter. Figure 3(a) shows the comparison between the excitonic absorption spectrum of the pristine and the SP- and MC-functionalized $(10,0)$ CNT. We observe (i) a redshift of the transition energy accompanied by a reduced intensity, and (ii) enhanced absorption at the higher energies for the functionalized CNT [see the insets of Fig. 3(a)]. In particular, the MC molecule with its large dipole moment (13.9 D) significantly influences the optical properties of the CNT leading to a redshift of $30 \mathrm{meV}$. In contrast, the SP molecule (6.2 D) only slightly changes the absorption spectrum of the substrate CNT (the redshift is about $5 \mathrm{meV}$ ). This clear difference between the spectra of MC- and SP-functionalized CNTs suggests the possibility of an optical readout of the SP-MC molecular switch. A study of other nanotubes with different chiral angles and diameters led to qualitatively similar results.

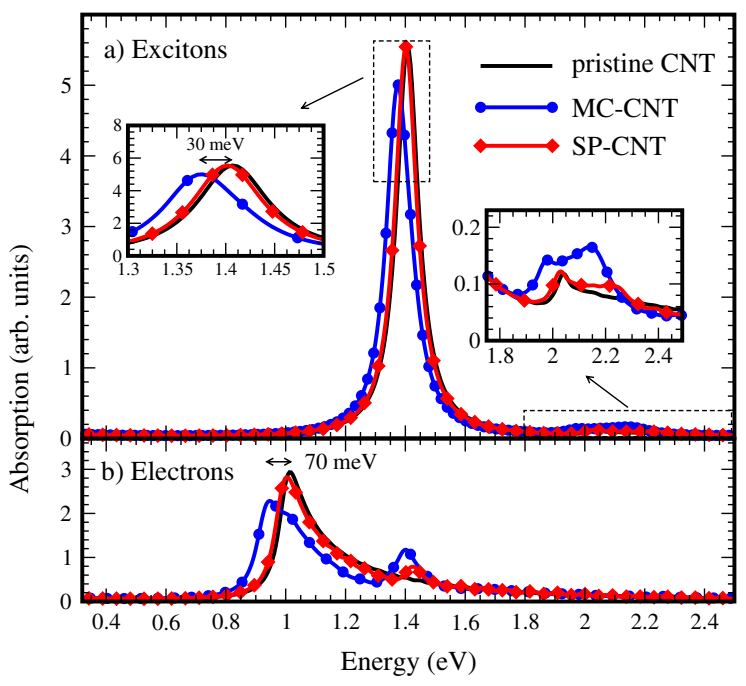

FIG. 3 (color online). Absorption spectrum of the pristine, SP-, and MC-functionalized $(10,0)$ nanotube within the (a) excitonic and (b) free-particle picture (with $n=0.25 \mathrm{~nm}^{-1}, \alpha=0^{\circ}$, and periodic distribution). Here, only the energetically lowest optical transition is taken into account. The insets illustrate the redshift of the transition energy and the enhanced absorption at higher energies. The more pronounced changes for the merocyanine molecule (MC) are due to its larger dipole moment.

Within the free-particle picture-important for CNTs in solutions with a large dielectric background constant- the influence of the functionalization is even more pronounced [Fig. 3(b)]. The redshift of the transition energy is enhanced to $70 \mathrm{meV}$ (10 meV) for MC-(SP-)functionalized CNTs. Furthermore, we observe the formation of a side peak at an energy of $1.4 \mathrm{eV}$. The observed changes in the absorption spectra of functionalized CNTs are similar to the polaronic redshift and the side peaks originating from the electron-phonon coupling [10]. We ascribe the smaller exciton-dipole coupling to the strong redistribution of the momentum via Coulomb interaction, which generates localized excitonic states.

To assure that the observed redshift of the transition energy is stable and to find optimal functionalization scenarios, we performed calculations for different dipole densities, distributions, and orientations [Fig. 4]. We observe that the rotation of the dipole with respect to the CNT axis has a significant influence on the observed redshift. Figure 4(a) shows that the largest shift of approximately $65 \mathrm{meV}$ can be found in excitonic absorption spectra in the case of perpendicularly oriented dipoles, i.e., $\alpha=90^{\circ}$ (in comparison, one obtains $30 \mathrm{meV}$ for $\alpha=0^{\circ}$ ). This reflects well the clearly stronger CNT-dipole matrix element $g_{k k^{\prime}}^{\lambda \lambda}$ in the perpendicular case [Fig. 2(a)]. For SP-functionalized CNTs, we observe negligibly small redshifts.

The molecule distribution along the CNT surface [Fig. 1] has been shown to play a role for the CNT-dipole coupling element and its multipeak structure [Fig. 2(c)]. However, it turns out to have no measurable influence on 

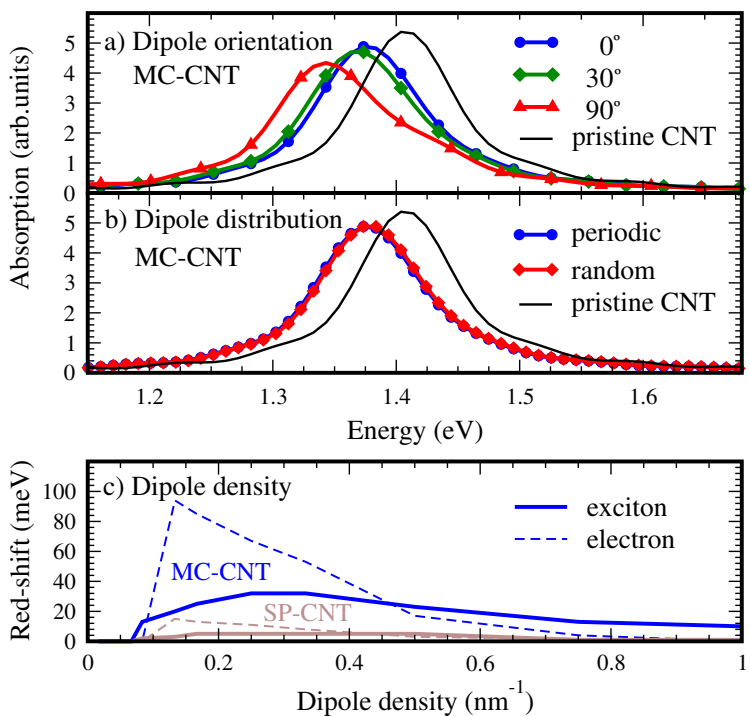

FIG. 4 (color online). Excitonic absorption spectrum of the pristine, MC-, and SP-functionalized $(10,0) \mathrm{CNT}$. The figure illustrates the influence of the functionalization depending on (a) the dipole orientation $\alpha$, (b) the dipole distribution, and (c) the dipole density $n$.

the peak positions and their redshift in the absorption spectra of functionalized CNTs [Fig. 4(b)]. The distribution only changes the enhancement of the absorption at higher energies; however, this effect is small in excitonic absorption spectra. The reason for this observation is that the redshift does not depend on the absolute values of the coupling element, but only on the integral over the momentum $k$ [Eq. (2)], which does not change with different distributions of molecules. The crucial quantity here is the dipole density. The more molecules are attached to the CNT surface, the larger the overall coupling is between the charge carriers within the CNT and the induced external dipole field. Therefore, one expects the influence on the optical properties of the substrate CNT to increase continuously as well. Figure 4(c), showing the redshift of the transition energy as a function of the dipole density $n$, suggests a more complicated behavior. We observe an enhanced redshift with an increasing dipole density of up to approximately $n=0.25 \mathrm{~nm}^{-1}$ followed by a decrease. This can be explained by the interference effects, which lead to the multipeak structure of the CNT-dipole coupling element [Fig. 2(b)]. Here, the peak separation is found to increase with the dipole density, i.e., the CNTdipole coupling becomes maximal for a very large momentum transfer $k$. Since the density of states is small for large $k$, the influence of the attached molecules on the optical properties of the CNT is reduced.

In summary, we investigated the optical properties of an exemplary hybrid structure consisting of a carbon nanotube as substrate and spiropyran as a photochrome molecule. We predict a redshift in both excitonic and free-particle absorption spectra of functionalized nanotubes due to an efficient coupling between the charge carriers and the externally switchable molecule dipole field. Depending on the conformation of the attached molecule, we find a redshift of the transition energy of up to $100 \mathrm{meV}$ suggesting that carbon nanotubes are suitable substrates for an unambiguous optical readout of spiropyran-based molecular switches. The gained insights about the coupling mechanism between the charge carriers in the one-dimensional nanosubstrate and the externally induced molecular dipole field can be applied to other hybrid structures.

We acknowledge financial support by the Deutsche Forschungsgemeinschaft through SFB 658. S. R. acknowledges funding by the ERC under Grant No. 210642.

*ermin.malic@tu-berlin.de

[1] A. Jorio, M. Dresselhaus, and G. Dresselhaus, Carbon Nanotubes: Advanced Topics in the Synthesis, Structure, Properties and Applications (Springer, Berlin, 2008).

[2] E. Del Canto, K. Flavin, M. Natali, T. Perova, and S. Giordani, Carbon 48, 2815 (2010).

[3] X. Zhou, T. Zifer, B. M. Wong, K. L. Krafcik, F. Leonard, and A. L. Vance, Nano Lett. 9, 1028 (2009).

[4] A. Hirsch and O. Vostrowsky, Top. Curr. Chem. 245, 193 (2005) http://www.springerlink.com/content/ j2gff1p1gn38nyk3.

[5] R. Khairutdinov, M. Itkis, and R. Haddon, Nano Lett. 4, 1529 (2004).

[6] X. Guo, L. Huang, S. O'Brien, P. Kim, and C. Nuckolls, J. Am. Chem. Soc. 127, 15045 (2005).

[7] G. Berkovic, V. Krongauz, and V. Weiss, Chem. Rev. 100, 1741 (2000).

[8] M. Del Valle, R. Gutirrez, C. Tejedor, and G. Cuniberti, Nature Nanotech. 2, 176 (2007).

[9] J. Whelan, D. Abdallah, J. Wojtyk, and E. Buncel, J. Mater. Chem. 20, 5727 (2010).

[10] H. Haug and S. W. Koch, Quantum Theory of the Optical and Electronic Properties of Semiconductors (World Scientific, Singapore, 2004).

[11] T. Winzer, A. Knorr, and E. Malic, Nano Lett. 10, 4839 (2010)

[12] M. J. Frisch, G.W. Trucks, and H. B. Schlegel et al., Gaussian 09 Revision A.1 (Gaussian Inc., Wallingford, Connecticut, 2009).

[13] E. Malić, M. Hirtschulz, F. Milde, A. Knorr, and S. Reich, Phys. Rev. B 74, 195431 (2006).

[14] M. Hirtschulz, F. Milde, E. Malić, S. Butscher, C. Thomsen, S. Reich, and A. Knorr, Phys. Rev. B 77, 035403 (2008).

[15] E. Malić, J. Maultzsch, S. Reich, and A. Knorr, Phys. Rev. B 82, 035433 (2010).

[16] J. Jiang, R. Saito, G. G. Samsonidze, A. Jorio, S. G. Chou, G. Dresselhaus, and M. S. Dresselhaus, Phys. Rev. B 75, 035407 (2007).

[17] E. Malić, M. Hirtschulz, J. Maultzsch, S. Reich, and A. Knorr, Phys. Status Solidi B 246, 2592 (2009). 